\title{
The force on a bubble, drop, or particle in arbitrary time-dependent motion at small Reynolds number
}

\author{
Phillip M. Lovalenti and John F. Brady \\ Department of Chemical Engineering 210-41, California Institute of Technology, Pasadena, California \\ 91125
}

(Received 1 December 1992; accepted 21 May 1993)

\begin{abstract}
The hydrodynamic force on a body that undergoes translational acceleration in an unbounded fluid at low Reynolds number is considered. The results extend the prior analysis of Lovalenti and Brady [to appear in J. Fluid Mech. (1993)] for rigid particles to drops and bubbles. Similar behavior is shown in that, with the inclusion of convective inertia, the long-time temporal decay of the force (or the approach to steady state) at finite Reynolds number is faster than the $t^{-1 / 2}$ predicted by the unsteady Stokes equations.
\end{abstract}

\section{INTRODUCTION}

In a recent paper, ${ }^{1}$ henceforth referred to as LB, the authors analyzed the force on a rigid particle in arbitrary time-dependent motion in a time-dependent uniform flow for small, but finite, Reynolds number, Re. The primary conclusion of that study was that the long-time temporal behavior of the hydrodynamic force decays faster than the $t^{-1 / 2}$ decay associated with the Basset history integral from unsteady Stokes flow. (For short time scale motion, however, the unsteady Stokes results are valid.) This change in the temporal decay for long time is the result of a transition in the mechanism of vorticity transport: from a symmetric diffusion of vorticity generated at the particle surface to convection of vorticity in the familiar Oseen wake behind the particle.

The motivation for extending the study to drops is to investigate the similarities and differences of the results for solid particles with those for drops and bubbles. Also, it is of value to have an expression for the unsteady force on a drop, which is useful in studies requiring the equation of motion of bubbles, drops, or particles at small but finite Reynolds number.

In what follows, we consider the hydrodynamic force for a drop in arbitrary time-dependent motion in an unbounded Newtonian fluid undergoing a time- and spatialdependent flow. This is accomplished through the use of the reciprocal theorem. We first derive the expression in general terms, and then simplify it for particular cases of drop composition, shape, and imposed flow. The results for spatially uniform flow are shown to follow directly from those for a rigid particle.

\section{RECIPROCAL THEOREM EXPRESSION FOR THE FORCE}

Consider a drop of density $\rho^{*}$ and viscosity $\mu^{*}$ in a fluid of density $\rho$ and viscosity $\mu$. Let $\lambda=\mu^{*} / \mu$ and $\beta=\left(v^{*} / v\right)^{1 / 2}$ where $v^{*}$ and $v$ are the kinematic viscosities of the drop and exterior fluid, respectively. The drop is translating with a time-dependent, center-of-mass velocity
$\overline{\mathbf{U}}(t)$ in an imposed flow $\mathbf{u}^{\infty}(\mathbf{x}, t)$. We begin by writing the Navier-Stokes equations for the fluids inside and outside the drop, where an asterisk (*) is used to denote variables and parameters associated with the interior fluid of the drop:

$$
\begin{aligned}
& \nabla \cdot \sigma^{*}=\rho^{*} \frac{D \mathbf{u}^{*}}{D t}, \quad \nabla \cdot \mathbf{u}^{*}=0 \text { inside the drop; } \\
& \nabla \cdot \sigma=\rho \frac{D \mathbf{u}}{D t}, \quad \nabla \cdot \mathbf{u}=0 \text { outside the drop. }
\end{aligned}
$$

Here, $\sigma=-p+\mu\left(\nabla \mathbf{u}+\nabla \mathbf{u}^{T}\right)$ is the stress tensor for a Newtonian fluid, and the pressure $p$ includes the effect of a uniform body force (e.g., gravity). Although the velocities are those relative to the fixed laboratory frame, the origin of the coordinate system is at the instantaneous center of mass of the drop, so that

$$
\frac{D \mathbf{u}}{D t}=\frac{\partial \mathbf{u}}{\partial t}+\mathbf{u} \cdot \nabla \mathbf{u}-\overline{\mathbf{U}}(t) \cdot \nabla \mathbf{u} .
$$

A position vector in this coordinate system will be denoted by $\mathbf{x}$.

If we assume immiscible fluids with constant surface tension $\gamma$, the appropriate boundary conditions at the interface of the drop and the exterior fluid are continuity of velocity and shear stress:

$$
\mathbf{u}=\mathbf{u}^{*}, \mathbf{n} \cdot\left(\boldsymbol{\sigma}-\boldsymbol{\sigma}^{*}\right) \cdot(\mathbf{I}-\mathbf{n n})=\mathbf{0} \text { on } S_{d},
$$

where $\mathbf{n}$ is the normal to the interface pointing into the exterior fluid and $S_{d}$ represents the surface of the drop. The second equation of (4) is also known as the tangential stress balance. In addition, the velocity normal to the interface may be given by

$$
\mathbf{n} \cdot \mathbf{u}=\mathbf{n} \cdot \mathbf{u}^{*}=\mathbf{n} \cdot \mathbf{U}\left(\mathbf{x}_{s}, t\right) \text { on } S_{d},
$$

where the velocity of the interface, $\mathbf{U}\left(\mathbf{x}_{s}, t\right)$ may be a func- 
tion of the position on the surface, $\mathbf{x}_{s}$. The conditions to be satisfied far from the drop are

$$
\mathbf{u} \rightarrow \mathbf{u}^{\infty}, p \rightarrow p^{\infty} \text { as } r \rightarrow \infty,
$$

where $r=|\mathbf{x}|$, and the imposed flow $\left(\mathbf{u}^{\infty}, p^{\infty}\right)$ satisfies the Navier-Stokes equations. Additionally, the velocity and pressure inside the drop are required to remain bounded.

To determine the drop shape the normal stress balance is also required:

$$
\left[\mathbf{n} \cdot\left(\boldsymbol{\sigma}-\boldsymbol{\sigma}^{*}\right)\right] \cdot \mathbf{n}=\gamma(\boldsymbol{\nabla} \cdot \mathbf{n})+\left(\mathbf{f}_{b}-\mathbf{f}_{b}^{*}\right) \cdot \mathbf{x} \text { on } S_{d}
$$

where $\mathrm{f}_{b}$ and $\mathrm{f}_{b}^{*}$ are the uniform body forces acting on the fluid exterior to and inside the drop, respectively, which are necessary here because they have been incorporated in the pressure term of the stress tensors. Although it does not directly influence the derivation that follows, the normal stress balance is included for completeness. For the low-Reynolds-number flows considered here, viscous forces dominate and the critical parameter determining the drop shape is the capillary number, $\mathrm{Ca}=\mu U_{d} / \gamma$, where $U_{c}$ is the characteristic velocity of the drop relative to the imposed flow. For unsteady Stokes flow conditions (in unsteady Stokes flow the convective terms of the NavierStokes equations [the last two terms of (3)] are neglected owing to the smallness of the Reynolds number while the time derivative in (3) is retained due to the unsteadiness of the flow), the spherical drop in a time-dependent uniform flow can be shown to be a shape which satisfies the governing equations and boundary conditions independent of $\mathrm{Ca}^{2}$ For small $\mathrm{Ca}$, the drop tends to remain spherical in the presence of a nonuniform flow or for finite $\mathrm{Re}$ conditions. The effect of a small but finite Reynolds number (i.e., the effect of the convective terms of the NavierStokes equations) on the deformation and drag of a translating drop has been studied by Taylor and Acrivos, ${ }^{3}$ although they identified the Weber number as the critical parameter that must be small to maintain a near spherical drop shape. The Weber number is equal to the product of $\mathrm{Ca}$ and $\mathrm{Re}$. The effect of a linear flow on the deformation of a drop for small Ca has been treated by Leal, ${ }^{4}$ which also has references to earlier works on drop deformation and breakup.

In order to make use of the reciprocal theorem for an unbounded domain, we require the disturbance quantities which decay at infinity. Thus, we define the following:

$$
\mathbf{u}^{\prime}=\mathbf{u}-\mathbf{u}^{\infty}, \quad p^{\prime}=p-p^{\infty}, \quad \boldsymbol{\sigma}^{\prime}=\boldsymbol{\sigma}-\boldsymbol{\sigma}^{\infty} .
$$

The governing equations for the disturbance fields are

$$
\boldsymbol{\nabla} \cdot \boldsymbol{\sigma}^{\prime}=\rho \frac{D \mathbf{u}^{\prime}}{D t}+\rho \boldsymbol{\nabla} \cdot\left(\mathbf{u}^{\prime} \mathbf{u}^{\infty}+\mathbf{u}^{\infty} \mathbf{u}^{\prime}\right), \quad \boldsymbol{\nabla} \cdot \mathbf{u}^{\prime}=0 ;
$$

and the boundary conditions become

$\mathbf{u}^{\prime}=\mathbf{u}^{*}-\mathbf{u}^{\infty}, \mathbf{n} \cdot\left(\boldsymbol{\sigma}^{\prime}+\sigma^{\infty}-\sigma^{*}\right) \cdot(\mathbf{I}-\mathbf{n n})=\mathbf{0}$ on $S_{d}$,

and

$$
\mathbf{u}^{\prime} \rightarrow 0, \quad \mathbf{p}^{\prime} \rightarrow 0 \text { as } r \rightarrow \infty \text {. }
$$

We shall also require the disturbance flow Stokes fields for the translating drop for use in the reciprocal theorem below. Denoting these fields with a caret ( ) ), the governing equations and boundary conditions are

$\boldsymbol{\nabla} \cdot \hat{\sigma}^{*}=\mathbf{0}, \quad \boldsymbol{\nabla} \cdot \hat{\mathbf{u}}^{*}=0 \quad$ inside the drop,

$\boldsymbol{\nabla} \cdot \hat{\boldsymbol{\sigma}}=\mathbf{0}, \quad \boldsymbol{\nabla} \cdot \hat{\mathbf{u}}=0 \quad$ outside the drop,

$\hat{\mathbf{u}}=\hat{\mathbf{u}}^{*}, \quad \mathbf{n} \cdot\left(\hat{\boldsymbol{\sigma}}-\hat{\boldsymbol{\sigma}}^{*}\right) \cdot(\mathbf{I}-\mathbf{n n})=\mathbf{0}, \quad \mathbf{n} \cdot \hat{\mathbf{u}}^{*}=\mathbf{n} \cdot \hat{\mathbf{U}} \quad$ on $S_{d}$,

where $\hat{\mathbf{U}}$ is a constant, and

$$
\hat{\mathbf{u}} \rightarrow 0 ; \hat{p} \rightarrow 0 \quad \text { as } r \rightarrow \infty .
$$

Using the velocity and stress fields defined above, the reciprocal theorems inside and outside the drop take the following form:

$$
\begin{gathered}
\int_{S_{d}}\left(\mathbf{n} \cdot \boldsymbol{\sigma}^{*}\right) \cdot \hat{\mathbf{u}}^{*} d S-\int_{V_{d}}\left(\nabla \cdot \boldsymbol{\sigma}^{*}\right) \cdot \hat{\mathbf{u}}^{*} d V \\
=\int_{S_{d}}\left(\mathbf{n} \cdot \hat{\boldsymbol{\sigma}}^{*}\right) \cdot \mathbf{u}^{*} d S
\end{gathered}
$$

and

$$
\begin{gathered}
\int_{S_{d}}\left(\mathbf{n} \cdot \boldsymbol{\sigma}^{\prime}\right) \cdot \hat{\mathbf{u}} d S+\int_{V_{f}}\left(\nabla \cdot \boldsymbol{\sigma}^{\prime}\right) \cdot \hat{\mathbf{u}} d V \\
=\int_{S_{d}}(\mathbf{n} \cdot \hat{\boldsymbol{\sigma}}) \cdot \mathbf{u}^{\prime} d S
\end{gathered}
$$

where we have assumed that by using disturbance quantities there is no contribution from the surface at infinity. (As discussed in LB, the requirement is that the disturbance pressure $p^{\prime}$ decays faster than $r^{-1}$, which is justified for the low-Reynolds-number flows to be considered here.) Here, $V_{d}$ and $V_{f}$ denote the volume of the drop and exterior fluid, respectively. Following a procedure similar to that used by Leal ${ }^{5}$ for bounded domains, we subtract (16) from (17), and, applying the boundary conditions (10) and (14) on the surface of the drop, obtain

$$
\begin{aligned}
& \int_{S_{d}} \mathbf{n} \cdot\left(\boldsymbol{\sigma}^{\prime}-\boldsymbol{\sigma}^{*}\right) \cdot \hat{\mathbf{u}}^{*} d S+\int_{V_{f}}\left(\nabla \cdot \boldsymbol{\sigma}^{\prime}\right) \cdot \hat{\mathbf{u}} d V \\
& \quad+\int_{V_{d}}\left(\nabla \cdot \boldsymbol{\sigma}^{*}\right) \cdot \hat{\mathbf{u}}^{*} d V \\
& \quad=\int_{S_{d}} \mathbf{n} \cdot\left(\hat{\boldsymbol{\sigma}}-\hat{\boldsymbol{\sigma}}^{*}\right) \cdot \mathbf{u}^{*} d S-\int_{S_{d}}(\mathbf{n} \cdot \hat{\boldsymbol{\sigma}}) \cdot \mathbf{u}^{\infty} d S .
\end{aligned}
$$

The first integral on the left-hand side (lhs) of (18) may be simplified by noting that 


$$
\begin{aligned}
\int_{S_{d}} \mathbf{n} \cdot\left(\boldsymbol{\sigma}^{\prime}-\boldsymbol{\sigma}^{*}\right) \cdot \hat{\mathbf{u}}^{*} d S & =\int_{S_{d}} \mathbf{n} \cdot\left(\boldsymbol{\sigma}-\boldsymbol{\sigma}^{*}\right) \cdot \hat{\mathbf{u}}^{*} d S-\int_{S_{d}} \mathbf{n} \cdot\left(\boldsymbol{\sigma}^{\infty}\right) \cdot \hat{\mathbf{u}}^{*} d S \\
& =\int_{S_{d}} \mathbf{n} \cdot\left(\boldsymbol{\sigma}-\boldsymbol{\sigma}^{*}\right) d S \cdot \hat{\mathbf{U}}-\int_{V_{d}}\left(\nabla \cdot \boldsymbol{\sigma}^{\infty}\right) \cdot \hat{\mathbf{u}}^{*} d V-\int_{V_{d}} \boldsymbol{\sigma}^{\infty}: \nabla \hat{\mathbf{u}}^{*} d V \\
& =\mathbf{F}_{d}^{H} \cdot \hat{\mathbf{U}}-\int_{V_{d}} \nabla \cdot \boldsymbol{\sigma}^{*} d V \cdot \hat{\mathbf{U}}-\int_{V_{d}}\left(\nabla \cdot \boldsymbol{\sigma}^{\infty}\right) \cdot \hat{\mathbf{u}}^{*} d V-\frac{1}{\lambda} \int_{S_{d}} \mathbf{n} \cdot \hat{\boldsymbol{\sigma}}^{*} \cdot \mathbf{u}^{\infty} d S
\end{aligned}
$$

where $\mathbf{F}_{d}^{H}\left(=\int_{S_{d}} \mathbf{n} \cdot \boldsymbol{\sigma} d S\right)$ is the total hydrodynamic force acting on the drop. The first equality is obtained simply by using the definition of $\sigma^{\prime}(8)$. The second equality is obtained by an application of the tangential (or shear) stress balance (4), the use of the drop surface boundary condition (14), and another application of the tangential stress balance, as follows:

$$
\begin{aligned}
\int_{S_{d}} \mathbf{n} \cdot\left(\boldsymbol{\sigma}-\boldsymbol{\sigma}^{*}\right) \cdot \hat{\mathbf{u}}^{*} d S \\
\quad=\int_{S_{d}} \mathbf{n} \cdot\left(\boldsymbol{\sigma}-\boldsymbol{\sigma}^{*}\right) \cdot[(\mathbf{I}-\mathbf{n n})+\mathbf{n n}] \cdot \hat{\mathbf{u}}^{*} d S \\
=\int_{S_{d}} \mathbf{n} \cdot\left(\boldsymbol{\sigma}-\boldsymbol{\sigma}^{*}\right) \cdot \mathbf{n n} \cdot \hat{\mathbf{u}}^{*} d S \\
=\int_{S_{d}} \mathbf{n} \cdot\left(\boldsymbol{\sigma}-\boldsymbol{\sigma}^{*}\right) \cdot \mathbf{n n} \cdot \hat{\mathbf{U}} d S \\
=\int_{S_{d}} \mathbf{n} \cdot\left(\boldsymbol{\sigma}-\boldsymbol{\sigma}^{*}\right) \cdot[(\mathbf{I}-\mathbf{n n})+\mathbf{n n}] d S \cdot \hat{\mathbf{U}} \\
=\int_{S_{d}} \mathbf{n} \cdot\left(\boldsymbol{\sigma}-\boldsymbol{\sigma}^{*}\right) d S \cdot \hat{\mathbf{U}} .
\end{aligned}
$$

The divergence theorem is also applied to obtain the two volume integrals in the second equality and the first volume integral in the last equality of (19). We note that if one makes use of the normal stress balance (7) in the third equality of (20), it will ultimately lead to an equation of motion for the drop instead of a derivation for the hydrodynamic force. This is the result of the fact that the first two terms in the last equality of (19), representing the total hydrodynamic force less the inertia of the drop, would be replaced by the negative of the external body forces acting on the drop. We will return to the derivation of the equation of motion of the drop in Sec. V. The very last integral of (19) was arrived at by the following series of steps:

$$
\int_{V_{d}} \sigma^{\infty}: \nabla \hat{\mathbf{u}}^{*} d V=\int_{V_{d}} \mu\left(\nabla \mathbf{u}^{\infty}+\nabla \mathbf{u}^{\infty} T\right): \nabla \hat{\mathbf{u}}^{*} d V
$$

(using the definition of a Newtonian fluid and that $p^{\infty} \mathbf{I}: \nabla \hat{\mathbf{u}}^{*}=0$ )

$$
\begin{aligned}
= & \int_{V_{d}} \mu\left(\nabla \mathbf{u}^{\infty}\right):\left(\nabla \hat{\mathbf{u}}^{*}+\nabla \hat{\mathbf{u}}^{* T}\right) d V \quad \text { (an identity) } \\
= & \frac{1}{\lambda} \int_{V_{d}} \nabla \mathbf{u}^{\infty}:\left[-\hat{p}^{*} \mathbf{I}+\mu^{*}\left(\nabla \hat{\mathbf{u}}^{*}+\nabla \hat{\mathbf{u}}^{* T}\right)\right] d V \\
& \left(\text { from } \hat{p}^{*} \mathbf{I}: \nabla \mathbf{u}^{\infty}=0\right) \\
= & \frac{1}{\lambda} \int_{V_{d}} \nabla \mathbf{u}^{\infty}: \hat{\boldsymbol{\sigma}}^{*} d V
\end{aligned}
$$

(using the definition of a Newtonian fluid)

$$
\begin{aligned}
& =\frac{1}{\lambda} \int_{V_{d}} \nabla \cdot\left(\hat{\boldsymbol{\sigma}}^{*} \cdot \mathbf{u}^{\infty}\right) d V \quad\left(\text { from } \boldsymbol{\nabla} \cdot \hat{\boldsymbol{\sigma}}^{*}=0\right) \\
& =\frac{1}{\lambda} \int_{S_{d}} \mathbf{n} \cdot \hat{\boldsymbol{\sigma}}^{*} \cdot \mathbf{u}^{\infty} d S,
\end{aligned}
$$

where the last step is obtained by applying the divergence theorem. In addition, the first integral of the right-hand side (rhs) of (18) may be reexpressed using the same steps as in (20) to obtain:

$$
\begin{aligned}
\int_{S_{d}} \mathbf{n} \cdot\left(\hat{\boldsymbol{\sigma}}-\hat{\boldsymbol{\sigma}}^{*}\right) \cdot \mathbf{u}^{*} d S \\
=\int_{S_{d}} \mathbf{n} \cdot\left(\hat{\boldsymbol{\sigma}}-\hat{\boldsymbol{\sigma}}^{*}\right) \cdot \mathbf{U}\left(\mathbf{x}_{s}, t\right) d S \\
=\int_{S_{d}} \mathbf{n} \cdot\left(\hat{\boldsymbol{\sigma}}-\hat{\boldsymbol{\sigma}}^{*}\right) \cdot \overline{\mathbf{U}}(t) d S \\
\quad+\int_{S_{d}} \mathbf{n} \cdot\left(\hat{\boldsymbol{\sigma}}-\hat{\boldsymbol{\sigma}}^{*}\right) \cdot \mathbf{U}^{\prime}\left(\mathbf{x}_{s}, t\right) d S \\
=\mathbf{F}_{d}^{H} \cdot \overline{\mathbf{U}}+\int_{S_{d}} \mathbf{n} \cdot\left(\hat{\boldsymbol{\sigma}}-\hat{\boldsymbol{\sigma}}^{*}\right) \cdot \mathbf{U}^{\prime}\left(\mathbf{x}_{s}, t\right) d S,
\end{aligned}
$$

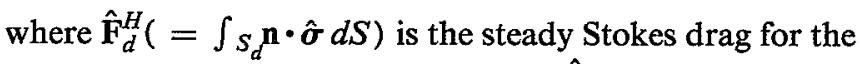
drop translating with velocity $\hat{\mathbf{U}}$, and $\mathbf{U}^{\prime}\left(\mathbf{x}_{s}, t\right)$ $\left[=\mathbf{U}\left(\mathbf{x}_{s}, t\right)-\overline{\mathbf{U}}(t)\right]$ is the velocity of the interface relative to that of the center of mass of the drop. To arrive at the last equality we have also used the fact that $\int_{S_{d} \mathbf{n}} \cdot \hat{\sigma}^{*} d S=0$ from an application of the divergence theorem. Combining (19) and (22) in (18) we have 


$$
\begin{aligned}
\mathbf{F}_{d}^{H} \cdot \hat{\mathbf{U}} & +\int_{V_{f}}\left(\nabla \cdot \boldsymbol{\sigma}^{\prime}\right) \cdot \hat{\mathbf{u}} d V+\int_{V_{d}}\left(\nabla \cdot \boldsymbol{\sigma}^{*}\right) \cdot\left(\hat{\mathbf{u}}^{*}-\hat{\mathbf{U}}\right) d V \\
& -\int_{V_{d}}\left(\boldsymbol{\nabla} \cdot \boldsymbol{\sigma}^{\infty}\right) \cdot \hat{\mathbf{u}}^{*} d V \\
= & \hat{\mathbf{F}}_{d}^{H} \cdot \overline{\mathbf{U}}-\int_{S_{d}}(\mathbf{n} \cdot \hat{\boldsymbol{\sigma}}) \cdot \mathbf{u}^{\infty} d S+\frac{1}{\lambda} \int_{S_{d}} \mathbf{n} \cdot \hat{\boldsymbol{\sigma}}^{*} \cdot \mathbf{u}^{\infty} d S \\
& +\int_{S_{d}} \mathbf{n} \cdot\left(\hat{\boldsymbol{\sigma}}-\hat{\boldsymbol{\sigma}}^{*}\right) \cdot \mathbf{U}^{\prime} d S .
\end{aligned}
$$

Noting that all the disturbance Stokes fields are linear in $\hat{\mathbf{U}}$, we define the following:

$$
\begin{array}{ll}
\hat{\mathbf{u}}=\hat{\mathbf{M}} \cdot \hat{\mathbf{U}}, & \hat{\mathbf{u}}^{*}=\hat{\mathbf{M}}^{*} \cdot \hat{\mathbf{U}}, \\
\hat{\boldsymbol{\sigma}}=\hat{\mathbf{T}} \cdot \hat{\mathbf{U}}, & \hat{\boldsymbol{\sigma}}^{*}=\hat{\mathbf{T}}^{*} \cdot \hat{\mathbf{U}},
\end{array}
$$

where $\hat{\mathbf{M}}$ and $\hat{\mathbf{M}}^{*}$ are second rank tensors and $\hat{\mathbf{T}}$ and $\hat{\mathbf{T}}^{*}$ are third rank tensors, all of which are functions of position. Also by linearity, the steady Stokes drag may be expressed as

$$
\hat{\mathbf{F}}_{d}^{H}=-\hat{\mathbf{R}}_{F U} \cdot \hat{\mathbf{U}}
$$

where $\hat{\mathbf{R}}_{F U}$ is the symmetric, second rank resistance tensor which is a function of the drop shape as well as the viscosity ratio $\lambda$. (Note that all the Stokes tensor quantities are evaluated at the current time, and thus may be a function of time if the drop is deforming.) Thus, since all terms of (23) are linear in an arbitrary vector, $\hat{\mathbf{U}}$, it may be eliminated from (23) to obtain

$$
\begin{aligned}
\mathbf{F}_{d}^{H}+ & \int_{V_{f}}\left(\nabla \cdot \boldsymbol{\sigma}^{\prime}\right) \cdot \hat{\mathbf{M}} d V+\int_{V_{d}}\left(\nabla \cdot \boldsymbol{\sigma}^{*}\right) \cdot\left(\hat{\mathbf{M}}^{*}-\mathbf{I}\right) d V \\
& -\int_{V_{d}}\left(\rho \frac{D \mathbf{u}^{\infty}}{D t}\right) \cdot \hat{\mathbf{M}}^{*} d V \\
& =-\mathbf{R}_{F U} \cdot \overline{\mathbf{U}}-\int_{S_{d}} \mathbf{u}^{\infty} \cdot(\mathbf{n} \cdot \hat{\mathbf{T}}) d S+\frac{1}{\lambda} \int_{S_{d}} \mathbf{u}^{\infty} \\
& \cdot\left(\mathbf{n} \cdot \hat{\mathbf{T}}^{*}\right) d S+\int_{S_{d}} \mathbf{U}^{\prime} \cdot\left[\mathbf{n} \cdot\left(\hat{\mathbf{T}}-\hat{\mathbf{T}}^{*}\right)\right] d S .
\end{aligned}
$$

Here $\mathbf{u}^{\infty}$ satisfies the Navier-Stokes equations and thus:

$$
\nabla \cdot \sigma^{\infty}=\rho \frac{D \mathbf{u}^{\infty}}{D t}=\rho\left(\frac{\partial \mathbf{u}^{\infty}}{\partial t}+\mathbf{u}^{\infty} \cdot \nabla \mathbf{u}^{\infty}-\overline{\mathbf{U}} \cdot \nabla \mathbf{u}^{\infty}\right) .
$$

Equation (26) is a general expression of the hydrodynamic force acting on a drop of arbitrary shape in an arbitrarily imposed flow, with, of course, the restriction that the particular drop shape satisfy the normal stress balance for the given imposed flow. Also, as yet, no restriction has been placed on the magnitude of the Reynolds number. The first volume integral on the lhs of (26) represents the inertial contributions to the force from the disturbance flow outside the drop. For a solid sphere under unsteady Stokes flow conditions it yields the familiar added mass and Basset force, which has been evaluated, for example, by Maxey and Riley. ${ }^{6}$ For small but finite Reynolds num- ber, this integral is also the origin of the Oseen correction ${ }^{7,8}$ for steady uniform flow and the Saffman lift force ${ }^{9}$ for steady simple shear flow. The second volume integral on the lhs of (26) is unique to a drop of finite viscosity, since, as will be shown in Sec. III, it is identically zero in the limit of a solid particle or a bubble (an inviscid drop). This term is necessary, however, to obtain the correct force expression for a drop of arbitrary viscosity; as shown in Sec. IV, it combines with the first integral to produce the unsteady Stokes force acting on a drop. The last integral on the lhs of (26) represents the contribution to the hydrodynamic force from the inertia of the imposed flow. The first two integrals on the rhs are those due to the viscous effects of the imposed flow which, as we shall see in Sec. III, lead to the Faxen-like corrections to the steady Stokes drag $-\hat{\mathbf{R}}_{F U} \cdot \overline{\mathbf{U}}$. The last integral is the contribution to the hydrodynamic force resulting from the drop changing shape with time.

\section{FURTHER SIMPLIFICATIONS OF THE RECIPROCAL THEOREM}

For a solid particle [in this case, $\mathbf{U}^{\prime}$ could represent solid body rotation, allowing the last integral of (26) to yield the contribution to the hydrodynamic force from, for example, a rotating, screw-shaped particle], $\hat{\mathbf{M}}^{*}=\mathbf{I}, \mathbf{U}^{\prime}=\mathbf{0}$, and $1 / \lambda \rightarrow 0$ so that $(26)$ becomes

$$
\begin{aligned}
\mathbf{F}_{p}^{H} & +\int_{V_{f}}\left(\nabla \cdot \sigma^{\prime}\right) \cdot \hat{\mathbf{M}} d V-\int_{V_{p}}\left(\rho \frac{D \mathbf{u}^{\infty}}{D t}\right) d V \\
& =-\hat{\mathbf{R}}_{F U} \cdot \overline{\mathbf{U}}-\int_{S_{p}} \mathbf{u}^{\infty} \cdot(\mathbf{n} \cdot \hat{\mathbf{T}}) d S .
\end{aligned}
$$

For a zero-viscosity bubble, $\lambda \rightarrow 0$ (i.e., $\mu^{*} \rightarrow 0$ for fixed $\mu)$ and $\hat{\mathbf{T}}^{*} \rightarrow 0$. [The quantity $\hat{\mathrm{T}}^{*}$ may actually tend to a constant associated with the pressure inside the bubble, but a constant tensor here does not affect the force expression (26).] Thus, Eq. (26) may be expressed as

$$
\begin{aligned}
\mathbf{F}_{b}^{H}+\int_{V_{f}}\left(\boldsymbol{\nabla} \cdot \boldsymbol{\sigma}^{\prime}\right) \cdot \hat{\mathbf{M}} d V-\int_{V_{b}}\left(\rho \frac{D \mathbf{u}^{\infty}}{D t}\right) \cdot \hat{\mathbf{M}}^{*} d V \\
=-\mathbf{R}_{F U} \cdot \overline{\mathbf{U}}-\int_{S_{b}} \mathbf{u}^{\infty} \cdot(\mathbf{n} \cdot \hat{\mathbf{T}}) d S \\
\\
+\int_{S_{b}} \mathbf{u}^{\infty} \cdot\left(\mathbf{n} \cdot \frac{1}{\lambda} \hat{\mathbf{T}}^{*}\right) d S+\int_{S_{b}} \mathbf{U}^{\prime} \cdot(\mathbf{n} \cdot \hat{\mathbf{T}}) d S,
\end{aligned}
$$

where the second integral of (26) was eliminated by noting the following: 


$$
\begin{aligned}
\int_{V_{b}}\left(\nabla \cdot \sigma^{*}\right) \cdot\left(\hat{\mathbf{M}}^{*}-\mathbf{I}\right) d V \\
=\int_{V_{b}}\left(-\nabla p^{*}\right) \cdot\left(\hat{\mathbf{M}}^{*}-\mathbf{I}\right) d V \\
=\int_{V_{b}}-\nabla \cdot\left[\left(\hat{\mathbf{M}}^{*}-\mathbf{I}\right) p^{*}\right] d V \\
\quad\left(\text { using } \nabla \cdot\left(\hat{\mathbf{M}}^{*}-\mathbf{I}\right)=0\right) \\
=\int_{S_{b}}-\mathbf{n} \cdot\left(\hat{\mathbf{M}}^{*}-\mathbf{I}\right) p^{*} d S
\end{aligned}
$$

(applying the divergence theorem)

$$
=\mathbf{0} \text {, }
$$

where the last step used the condition that $\mathbf{n} \cdot \hat{\mathbf{M}}^{*}=\mathbf{n} \cdot \mathbf{I}$ on the bubble surface. The second and fourth integrals of (29) are evaluated in the limit as $\mu^{*} \rightarrow 0$. Alternatively, one can replace these two integrals with their original form, $\int_{S_{b}}\left(\mathbf{n} \cdot \sigma^{\infty}\right) \cdot \hat{\mathbf{M}} d S$, on the rhs of (29), although this is not explicit in $\mathbf{u}^{\infty}$. Note also that the fourth integral of (29) is a bounded quantity since $\hat{\mathbf{T}}^{*}$ scales linearly with $\mu^{*}$ and thus $(1 / \lambda) \hat{\mathrm{T}}^{*}$ scales with $\mu$, independent of $\mu^{*}$.

In the case of a spherical drop, the tensors associated with the disturbance Stokes flow problem are known from the Hadamard-Rybczyński solution of (12)-(15) with (24) and (25). They are given by

$$
\begin{aligned}
& \hat{\mathbf{M}} *=\frac{1}{2(\lambda+1)}\left[\left(2 \lambda+3-2 \frac{r^{2}}{a^{2}}\right) \mathbf{I}+\frac{\mathbf{x x}}{a^{2}}\right] \\
& \hat{\mathbf{M}}=\frac{3 \lambda+2}{4(\lambda+1)} \frac{a}{r}\left(\mathbf{I}+\frac{\mathbf{x x}}{r^{2}}\right)+\frac{\lambda}{4(\lambda+1)} \frac{a^{3}}{r^{3}}\left(\mathbf{I}-3 \frac{\mathbf{x x}}{r^{2}}\right) \\
& \left.\mathbf{n} \cdot \hat{\mathbf{T}}\right|_{r=a}=-\frac{3 \mu^{*}}{2 a(\lambda+1)} \mathbf{I}-\frac{6 \mu}{2 a(\lambda+1)} \mathbf{n n} \\
& \left.\mathbf{n} \cdot \hat{\mathbf{T}}^{*}\right|_{r=a}=-\frac{3 \mu^{*}}{2 a(\lambda+1)} \mathbf{I}+\frac{9 \mu^{*}}{2 a(\lambda+1)} \mathbf{n n}
\end{aligned}
$$

and

$$
\hat{\mathbf{R}}_{F U}=6 \pi \mu a\left(\frac{\lambda+2 / 3}{\lambda+1}\right) \mathbf{I},
$$

where $a$ is the radius of the drop. Thus, (26) may be expressed for a spherical drop as

$$
\begin{aligned}
\mathbf{F}_{d}^{H}+ & \int_{V_{f}}\left(\nabla \cdot \sigma^{\prime}\right) \cdot \hat{\mathbf{M}} d V+\int_{V_{d}}\left(\nabla \cdot \sigma^{*}\right) \cdot\left(\hat{\mathbf{M}}^{*}-\mathbf{I}\right) d V \\
& -\int_{V_{d}}\left(\rho \frac{D \mathbf{u}^{\infty}}{D t}\right) \cdot \hat{\mathbf{M}}^{*} d V \\
= & -6 \pi \mu a\left(\frac{\lambda+2 / 3}{\lambda+1}\right) \overline{\mathbf{U}}+\frac{3 \mu}{2 a}\left(\frac{\lambda-1}{\lambda+1}\right) \int_{S_{d}} \mathbf{u}^{\infty} d S \\
& +\frac{15 \mu}{2 a^{2}(\lambda+1)} \int_{V_{d}} \mathbf{u}^{\infty} d V
\end{aligned}
$$

where we have used the fact that for a sphere $\int_{S_{d}} \mathbf{u}^{\infty} \cdot \mathbf{n n} d S=\int V_{d}^{\mathbf{u}^{\infty}} d V$. The last integral of (26) is zero because the drop shape is fixed (or because $\mathbf{U}^{\prime}$ has zero center-of-mass velocity).

To simplify (36) further, we can express $\mathbf{u}^{\infty}$ and $D u^{\infty} / D t$ as multipole expansions about the center of mass of the drop, assuming the variation of the imposed flow is small over the dimensions of the drop:

$$
\begin{aligned}
\mathbf{u}^{\infty}(\mathbf{x}, t)=\mathbf{U}^{\infty}(t)+\mathbf{x} \cdot \nabla \mathbf{u}^{\infty}+\frac{\mathbf{x x}}{2 !} \cdot \nabla\left(\nabla \mathbf{u}^{\infty}\right)+\cdots, & \\
\frac{D \mathbf{u}^{\infty}}{D t}(\mathbf{x}, t)= & \frac{D \mathbf{u}^{\infty}}{D t}(0, t)+\mathbf{x} \cdot \nabla \frac{D \mathbf{u}^{\infty}}{D t}+\frac{\mathbf{x x}}{2 !}: \nabla\left(\nabla \frac{D \mathbf{u}^{\infty}}{D t}\right) \\
& +\cdots,
\end{aligned}
$$

where $\mathbf{U}^{\infty}(t)=\mathbf{u}^{\infty}(0, t)$ and the higher-order derivatives are evaluated at the instantaneous center of mass of the drop at time $t$. Using (37) and (38) in (36) and retaining terms up to those including quadratic variations in $\mathbf{u}^{\infty}$, we have for a spherical drop

$$
\begin{aligned}
\mathbf{F}_{d}^{H} & +\int_{V_{f}}\left(\nabla \cdot \sigma^{\prime}\right) \cdot \hat{\mathbf{M}} d V+\int_{V_{d}}\left(\nabla \cdot \sigma^{*}\right) \cdot\left(\hat{\mathbf{M}}^{*}-\mathbf{I}\right) d V \\
& -\left.\frac{4 \pi}{3} a^{3} \rho\left[\frac{D \mathbf{u}^{\infty}}{D t}+\left(\frac{\lambda-1 / 2}{\lambda+1}\right) \frac{a^{2}}{10} \nabla^{2} \frac{D \mathbf{u}^{\infty}}{D t}\right]\right|_{\mathbf{x}=0} \\
& =-6 \pi \mu a\left(\frac{\lambda+2 / 3}{\lambda+1}\right)\left(\overline{\mathbf{U}}-\mathbf{U}^{\infty}-\left.\frac{3 \lambda}{3 \lambda+2} \frac{a^{2}}{6} \nabla^{2} \mathbf{u}^{\infty}\right|_{\mathbf{x}=0}\right),
\end{aligned}
$$

where we have used the following equalities to show the two forms of the quadratic variation in $D u^{\infty} / D t$ are equivalent up to quadratic variations in $\mathbf{u}^{\infty}$ :

$$
\begin{aligned}
& \nabla^{2} \frac{D \mathbf{u}^{\infty}}{D t}=\frac{1}{\rho} \nabla^{2}\left(-\nabla \rho^{\infty}+\mu \nabla^{2} \mathbf{u}^{\infty}\right) ; \\
& \nabla \nabla \cdot \frac{D \mathbf{u}^{\infty}}{D t}=-\frac{1}{\rho} \nabla \nabla^{2} p^{\infty},
\end{aligned}
$$

where in (41) we have used the condition that $\nabla \cdot \mathbf{u}^{\infty}=0$.

\section{THE FORCE ACTING ON A DROP TRANSLATING IN A UNIFORM FLOW AT SMALL REYNOLDS NUMBER}

To evaluate precisely the first two integrals of the generalized expression for the hydrodynamic force, (26), would require the solution to the full Navier-Stokes equations for the translating drop. Although we shall not attempt to solve them in general, we can make some progress for the condition of a uniform, time-dependent imposed flow, when $\mathbf{u}^{\infty}=\mathbf{U}^{\infty}(t)$.

For uniform flow and arbitrary, but fixed drop shape (a condition generally satisfied if $\mathrm{Ca}<1$ ), (26) becomes 


$$
\begin{aligned}
\mathbf{F}_{d}^{H} & +\int_{V_{f}}\left(\boldsymbol{\nabla} \cdot \boldsymbol{\sigma}^{\prime}\right) \cdot \hat{\mathbf{M}} d V+\int_{V_{d}}\left(\nabla \cdot \boldsymbol{\sigma}^{*}\right) \cdot\left(\hat{\mathbf{M}}^{*}-\mathbf{I}\right) d V \\
& -\rho V_{d} \dot{\mathbf{U}}^{\infty}(t)=-\hat{\mathbf{R}}_{F U} \cdot \overline{\mathbf{U}}_{s}(t)
\end{aligned}
$$

where $\overline{\mathbf{U}}_{s}(t)\left[=\overline{\mathbf{U}}(t)-\mathbf{U}^{\infty}(t)\right]$ is the slip velocity of the drop. Here we have used the fact that the first two integrals on the rhs of (26) may be simplified by noting $\int_{S_{d}}(\mathbf{n} \cdot \hat{\mathbf{T}}) d S=-\hat{\mathbf{R}}_{F U}$ and $\int_{S_{d}}\left(\mathbf{n} \cdot \hat{\mathbf{T}}^{*}\right) d S=\mathbf{0}$. The goal now is to estimate the contributions from the two integrals in (42) with the condition that the Reynolds number $\left(\operatorname{Re}=a U_{d} v\right)$ for the fluid inside and outside the drop, based on the drop's slip velocity, is small but finite. (For a nonspherical body, $a$ denotes the characteristic drop dimension; otherwise it is the drop radius.) In so doing, we will obtain an expression for the hydrodynamic force acting on the drop to $O(\operatorname{Re})$ for arbitrary time-dependent motion.

First note the following equalities for the fluid exterior to the drop:

$\mathbf{f}^{\prime} \equiv \rho\left(\frac{\partial \mathbf{u}^{\prime}}{\partial t}-\overline{\mathbf{U}}_{s} \cdot \nabla \mathbf{u}^{\prime}+\mathbf{u}^{\prime} \cdot \nabla \mathbf{u}^{\prime}\right)=-\nabla p^{\prime}+\mu \nabla^{2} \mathbf{u}^{\prime}=\nabla \cdot \sigma^{\prime}$.

And if we define

$$
p^{* \prime}=p^{*}-\frac{\rho^{*}}{\rho} p^{\infty}, \quad \mathbf{u}^{* \prime}=\mathbf{u}^{*}-\mathbf{U}^{\infty}(t),
$$

we can note the following equalities for the fluid inside the drop:

$$
\begin{aligned}
\mathbf{f}^{* \prime} & \equiv \rho^{*}\left(\frac{\partial \mathbf{u}^{* \prime}}{\partial t}-\overline{\mathbf{U}}_{s} \cdot \nabla \mathbf{u}^{* \prime}+\mathbf{u}^{* \prime} \cdot \nabla \mathbf{u}^{* \prime}\right) \\
& =-\nabla p^{* \prime}+\mu^{*} \nabla^{2} \mathbf{u}^{* \prime}=\nabla \cdot \sigma^{*}-\rho^{*} \dot{\mathbf{U}}^{\infty}(t),
\end{aligned}
$$

where we have applied the condition that $-\left(\rho^{*} / \rho\right) \nabla p^{\infty}=\rho^{*} \dot{\mathbf{U}}^{\infty}(t)$. Using $\mathbf{f}^{\prime}$ and $\mathbf{f}^{* \prime}$ to signify the inertial terms from the first equalities of (43) and (45), the hydrodynamic force can now be expressed as

$$
\begin{aligned}
\mathbf{F}_{d}^{H}-\rho V_{d} \dot{\mathbf{U}}^{\infty}(t)= & -\hat{\mathbf{R}}_{F U} \cdot \overline{\mathbf{U}}_{s}(t)-\int_{V_{f}} \mathbf{f}^{\prime} \cdot \hat{\mathbf{M}} d V \\
& -\int_{V_{d}} \mathbf{f}^{* \prime} \cdot\left(\hat{\mathbf{M}}^{*}-\mathbf{I}\right) d V .
\end{aligned}
$$

Here we have used arguments similar to (30) to show

$$
\begin{gathered}
\int_{V_{d}}\left(\nabla \cdot \sigma^{*}\right) \cdot\left(\hat{\mathbf{M}}^{*}-\mathbf{I}\right) d V-\int_{V_{d}} \mathbf{f}^{* \prime} \cdot\left(\hat{\mathbf{M}}^{*}-\mathbf{I}\right) d V \\
=\rho^{*} \dot{\mathbf{U}}^{\infty}(t) \cdot \int_{V_{d}}\left(\hat{\mathbf{M}}^{*}-\mathbf{I}\right) d V \\
=\rho^{*} \dot{\mathbf{U}}^{\infty}(t) \cdot \int_{V_{d}} \nabla \cdot\left[\left(\hat{\mathbf{M}}^{*}-\mathbf{I}\right) \mathbf{r}\right] d V \\
=\rho^{*} \dot{\mathbf{U}}^{\infty}(t) \cdot \int_{S_{d}} \mathbf{n} \cdot\left[\left(\hat{\mathbf{M}}^{*}-\mathbf{I}\right) \mathbf{r}\right] d S=\mathbf{0} .
\end{gathered}
$$

Now since the boundary conditions for the "primed" fields are

$$
\begin{aligned}
& \mathbf{n} \cdot\left[\mu\left(\nabla \mathbf{u}^{\prime}+\nabla \mathbf{u}^{\prime T}\right)-\mu^{*}\left(\nabla \mathbf{u}^{* \prime}+\nabla \mathbf{u}^{* \prime T}\right)\right] \cdot(\mathbf{I}-\mathbf{n n})=\mathbf{0} \\
& \mathbf{u}^{\prime}=\mathbf{u}^{* \prime}, \quad \mathbf{n} \cdot \mathbf{u}^{\prime}=\mathbf{n} \cdot \mathbf{u}^{* \prime}=\mathbf{n} \cdot \overline{\mathbf{U}}_{s} \text { on } S_{d}
\end{aligned}
$$

and

$$
\mathbf{u}^{\prime} \rightarrow 0, \quad p^{\prime} \rightarrow 0 \quad \text { as } r \rightarrow \infty
$$

it can be seen that the two volume integrals in (46) represent the inertial corrections to the steady Stokes drag for a drop translating with velocity $\overline{\mathbf{U}}_{s}$ in a quiescent fluid. In addition, other than the presence of the integral over the volume of the drop, (46) is identical to the expression for a solid particle. Thus, with appropriate modifications, we can make use of the results for solid particles from LB. We will summarize the general ideas from that paper to show the similarities and differences with the current derivation. The interested reader is referred to the original work for further details.

For small Reynolds number and short time scale motion $\left(\tau_{c}<v / U_{c}^{2}\right.$ where $\tau_{c}$ is the time scale for the change in the drops slip velocity), the flow is governed, to leading order in $\mathrm{Re}$, by the unsteady Stokes equations throughout the fluid domain:

$$
-\nabla p^{\prime}+\mu \nabla^{2} \mathbf{u}^{\prime}=\rho \frac{\partial \mathbf{u}^{\prime}}{\partial t}, \quad \nabla \cdot \mathbf{u}^{\prime}=0 .
$$

This approximation is appropriate for the flow inside as well as outside the drop. The convective terms of the Navier-Stokes equations, $\mathbf{u} \cdot \nabla \mathbf{u}$ and $\overline{\mathbf{U}}_{s} \cdot \nabla \mathbf{u}$, are everywhere smaller than the viscous or the unsteady inertial terms, because the vorticity produced at the surface of the drop has not diffused out to the Oseen distance, $v / U_{c}$, where convection becomes important as a transport mechanism. Under these conditions, the contributions from the convective terms are obtained solely from a regular perturbation analysis.

On the other hand, for long time scale motion $\left(\tau_{c} \geqslant v / U_{c}^{2}\right)$ the flow in the near-field region (for length scales shorter than the Oseen distance $v / v_{c}$ ) is governed by the steady Stokes equations, while that in the far field [defined by distances from the drop of $O\left(v / U_{c}\right)$ or greater] is determined by the unsteady Oseen equations to leading order:

$$
\begin{aligned}
& -\nabla p^{\prime}+\mu \nabla^{2} \mathbf{u}^{\prime}=\rho\left(\frac{\partial \mathbf{u}^{\prime}}{\partial t}-\overline{\mathbf{U}}_{s} \cdot \nabla \mathbf{u}^{\prime}\right)+\mathbf{F}_{\mathrm{St}}^{H} \delta(\mathbf{x}), \\
& \nabla \cdot \mathbf{u}^{\prime}=0 .
\end{aligned}
$$

Here, the boundary conditions at the drop surface are replaced by the presence of the force monopole in the governing equation; that is, to leading order in the far-field region, the particle appears as a point-force disturbance of magnitude the pseudosteady Stokes drag $\mathbf{F}_{\mathbf{S t}}^{H}$ $\left[=-\hat{\mathbf{R}}_{F U} \cdot \overline{\mathbf{U}}_{s}(t)\right]$. Also, in this case, diffusion and convection are of equal importance in the transport of vorticity.

For motion of arbitrary time scale, the unsteady Stokes equations describe the flow to leading order everywhere, except in the far field where the unsteady Oseen equations 
govern the flow when the time scale of the motion is large. Thus, in evaluating the volume integrals of (46), one is able to identify three sources of inertial terms that can contribute to the hydrodynamic force to $O(\operatorname{Re})$ : those from unsteady Stokes flow, those from applying regular perturbation techniques to the unsteady Stokes equations in accounting for the convective terms, and those from unsteady Oseen flow. After taking the proper precautions to prevent a double-counting of contributions from these sources, one arrives at the following expression for the hydrodynamic force acting on the drop (basically by analogy with the results from $\mathrm{LB}$ ):

$\mathbf{F}_{d}^{H}-\rho V_{d} \dot{\mathbf{U}}^{\infty}(t)$

$$
\begin{aligned}
= & \mathbf{F}_{U \mathrm{St}}^{H}-\int_{V_{f}} \rho\left(\frac{\partial \mathbf{u}_{1}^{\prime}}{\partial t}-\overline{\mathbf{U}}_{s} \cdot \nabla \mathbf{u}_{0}^{\prime}+\mathbf{u}_{0}^{\prime} \cdot \nabla \mathbf{u}_{0}^{\prime}\right) \cdot \hat{\mathbf{M}} d V \\
& -\int_{V_{d}} \rho^{*}\left(\frac{\partial \mathbf{u}_{1}^{* \prime}}{\partial t}-\overline{\mathbf{U}}_{s} \cdot \nabla \mathbf{u}_{0}^{* \prime}+\mathbf{u}_{0}^{* \prime} \cdot \nabla \mathbf{u}_{0}^{* \prime}\right) \cdot \hat{\mathbf{M}}^{*} d V \\
& -\sqrt{\frac{a^{2}}{\pi v}} \int_{-\infty}^{t} \frac{\dot{\mathbf{F}}_{\mathrm{St}}^{H}(s)}{\sqrt{t-s}} d s \cdot \Phi+\mathbf{F}_{\mathrm{Os}}^{H} .
\end{aligned}
$$

This expression retains the leading-order effects of the convective inertia of the fluid for small $\mathrm{Re}$, accurate to $O\left(\mu a U_{c} \operatorname{Re}\right)$. The quantity $F_{U S \mathrm{t}}^{H}$, henceforth referred to as the unsteady Stokes force, represents the hydrodynamic force acting the drop translating with velocity $\overline{\mathbf{U}}_{s}(t)$ in a quiescent fluid as determined by the unsteady Stokes equations (49).

The two volume integrals of (51) are from the regular perturbation to unsteady Stokes flow. The velocity fields $\mathbf{u}_{0}^{\prime}$ and $\mathbf{u}_{0}^{* \prime}$ are the solutions to (49) with the boundary conditions given by (48). The velocity fields $\mathbf{u}_{1}^{\prime}$ and $\mathbf{u}_{1}^{* \prime}$ are the regular perturbation to unsteady Stokes flow for convection. They satisfy

$$
\begin{aligned}
& -\nabla p_{1}^{\prime}+\mu \nabla^{2} \mathbf{u}_{1}^{\prime}=\rho\left(\frac{\partial \mathbf{u}_{1}^{\prime}}{\partial t}-\overline{\mathbf{U}}_{s} \cdot \nabla \mathbf{u}_{0}^{\prime}+\mathbf{u}_{0}^{\prime} \cdot \nabla \mathbf{u}_{0}^{\prime}\right), \\
& \nabla \cdot \mathbf{u}_{1}^{\prime}=0,
\end{aligned}
$$

for $\mathbf{u}_{1}^{\prime}$ and the same equations for $\mathbf{u}_{1}^{* \prime}$ by replacing all quantities in (52) with those corresponding to the fluid in the drop, which are denoted by an asterisk. The boundary conditions are

$$
\begin{aligned}
& \mathbf{n} \cdot\left[\mu\left(\nabla \mathbf{u}_{1}^{\prime}+\nabla \mathbf{u}_{1}^{\prime T}\right)-\mu^{*}\left(\nabla \mathbf{u}_{1}^{* \prime}+\nabla \mathbf{u}_{1}^{* \prime T}\right)\right] \cdot(\mathbf{I}-\mathbf{n n})=\mathbf{0}, \\
& \mathbf{u}_{1}^{\prime}=\mathbf{u}_{1}^{* \prime}, \quad \mathbf{n} \cdot \mathbf{u}_{1}^{\prime}=\mathbf{n} \cdot \mathbf{u}_{1}^{* \prime}=0 \text { on } S_{d} .
\end{aligned}
$$

The last two terms of (51) are attributed to the unsteady Oseen flow, the first of which is the negative of the longtime asymptotic form of the history force from unsteady Stokes flow, where the second rank tensor $\boldsymbol{\Phi}$ is defined by

$$
\boldsymbol{\Phi}=\frac{\hat{\mathbf{R}}_{F U}}{6 \pi \mu a} .
$$

The last term $F_{\mathrm{Os}}^{H}$, referred to as the unsteady Oseen force, is a new history integral which can be expressed by

$$
\begin{aligned}
\mathbf{F}_{\mathrm{Os}}^{H}(t)= & \frac{3}{8} \sqrt{\frac{a^{2}}{\pi v}}\left(\int _ { - \infty } ^ { t } \left[\frac{2}{3} \mathbf{F}_{\mathrm{St}}^{H \|}(t)-\left[\frac { 1 } { A ^ { 2 } } \left(\frac{\sqrt{\pi}}{2 A} \operatorname{erf}(A)\right.\right.\right.\right. \\
& \left.\left.-\exp \left(-A^{2}\right)\right)\right] \mathbf{F}_{\mathrm{St}}^{H \|}(s)+\frac{2}{3} \mathbf{F}_{\mathrm{St}}^{H \perp}(t) \\
& -\left[\exp \left(-A^{2}\right)-\frac{1}{2 A^{2}}\left(\frac{\sqrt{\pi}}{2 A} \operatorname{erf}(A)\right.\right. \\
& \left.\left.\left.\left.-\exp \left(-A^{2}\right)\right)\right] \mathbf{F}_{\mathrm{St}}^{H \perp}(s)\right\} \frac{2 d s}{(t-s)^{3 / 2}}\right) \cdot \boldsymbol{\Phi} .
\end{aligned}
$$

Here, $A$ has the definition

$$
A=\frac{1}{2} \sqrt{\frac{t-s}{v}}\left(\frac{\left|\mathbf{Y}_{s}(t)-\mathbf{Y}_{s}(s)\right|}{t-s}\right),
$$

where the displacement vector, $\mathbf{Y}_{s}(t)-\mathbf{Y}_{s}(s)$, is the time integration of $\overline{\mathbf{U}}_{s}$ from $s$ to $t$. The quantities $\mathbf{F}_{\mathrm{St}}^{H \|}$ and $\mathbf{F}_{S t}^{H \perp}$ are the components of the pseudosteady Stokes force $\mathbf{F}_{\mathrm{St}}^{H}$ parallel and perpendicular to this displacement vector. For short time scale motion $\left(\left\langle v / U_{c}^{2}\right), \mathbf{F}_{\mathrm{O}}^{H}\right.$ behaves as the negative of the history integral in (51) so that their combined contribution to the hydrodynamic force is smaller than $O\left(\mu a U_{c} \operatorname{Re}\right)$. For long time scale motion on the other hand, the history-dependent part of $F_{U S t}^{H}$ will cancel with this history integral in (51), and the dominant history dependence of the hydrodynamic force comes from $\mathbf{F}_{\mathrm{O} \text { s }}^{H}$.

If one has the unsteady Stokes solution for the translating drop, (51) can be used to obtain a closed-form expression for the hydrodynamic force for small but finite Reynolds number. In the case of a spherical drop, for example, the analysis is simplified by the fact that the contributions from the regular perturbation to unsteady Stokes flow [the two volume integrals of (51)] are identically zero, as can be seen from a symmetry argument. The unsteady Stokes force for a spherical drop in the frequency domain has been analyzed by Kim and Karrila. ${ }^{10}$ Their result implicitly assumes the kinematic viscosities of the fluid inside and outside the drop are equal, but this is easily generalized to arbitrary kinematic viscosity ratios; the corrected result as a function of the frequency $\omega$ is

$$
\begin{aligned}
\widetilde{\mathbf{F}}_{U S \mathrm{t}}^{H}(\omega)= & -6 \pi \mu a \widetilde{\mathbf{U}}_{s}(\omega)\left(1+\alpha+\frac{\alpha^{2}}{9}\right. \\
& \left.-\frac{(1+\alpha)^{2} f(\alpha \beta)}{\lambda g(\alpha \beta)+(3+\alpha) f(\alpha \beta)}\right),
\end{aligned}
$$

where

$$
f(\alpha)=\alpha^{2} \tanh \alpha-3 \alpha+3 \tanh \alpha,
$$

and

$$
g(\alpha)=\alpha^{3}+6 \alpha-6 \tanh \alpha-3 \alpha^{2} \tanh \alpha .
$$

Here $\alpha=\sqrt{-i \omega a^{2} / v}$ is the dimensionless frequency parameter and $\beta=\sqrt{v / v^{*}}$. The primary result of (57) is that the history integral for unsteady Stokes flow for a drop is not of the same form as that for a solid sphere because of 
the fourth term of the expression, which vanishes in the case of a solid. Although the resulting memory kernel continues to behave as $t^{-1 / 2}$ in both the limit as $t \rightarrow 0$ and as $t \rightarrow \infty$, the coefficient of the $t^{-1 / 2}$ term is different in the long-time asymptotic expression from that for short time scales $\left(t<a^{2} / v\right)$, except for a bubble, which tends toward a constant as $t \rightarrow 0 .{ }^{11}$

For a spherical bubble (57) simplifies considerably, allowing one to obtain a closed-form expression for the unsteady Stokes force for motion of arbitrary time scale. Using the analytical result from Yang and Leal ${ }^{11}$ for the unsteady Stokes force, (51) for a spherical bubble becomes

$$
\begin{aligned}
\mathbf{F}_{b}^{H} & -\frac{4 \pi}{3} a^{3} \rho \dot{\mathbf{U}}^{\infty}(t) \\
& =-4 \pi \mu a \overline{\mathbf{U}}_{s}(t)-\frac{2}{3} \pi \rho a^{3} \overline{\mathbf{U}}_{s}(t)-8 \pi \mu a
\end{aligned}
$$

$$
\begin{aligned}
& \times \int_{-\infty}^{t} e^{9 v(t-s) / a^{2}} \operatorname{erfc}\left[\sqrt{9 v(t-s) / a^{2}}\right] \dot{\mathbf{U}}_{s}(s) d s \\
& +\frac{8 \pi \mu a}{\sqrt{\pi}} \int_{-\infty}^{t} \frac{\dot{\mathbf{U}}_{s}(s)}{\sqrt{9 v(t-s) / a^{2}}} d s+F_{\mathrm{Os}}^{H},
\end{aligned}
$$

where in (55), the expression for $\mathbf{F}_{\mathrm{Os}}^{H}, \mathbf{F}_{\mathrm{St}}^{H}(t)$ is replaced by $-4 \pi \mu a \overline{\mathbf{U}}_{s}(t)$ and $\Phi$ by $\frac{2}{3} \mathbf{I}$. By an asymptotic analysis it can be seen that the memory kernel of the first integral of (60) tends to a constant for small time and behaves as the negative of the second memory function for large time.

For a drop of arbitrary shape, one can obtain an expression for the hydrodynamic force in terms of the results from steady Stokes flow in the limit when the time scale $\tau_{c}$ of the variation of the drop's slip velocity satisfies $\tau_{c} \gg a^{2} / v$. Following the procedure from LB, we obtain

$$
\begin{aligned}
\mathbf{F}_{d}^{H}(t)= & \rho V_{d} \dot{\mathbf{U}}^{\infty}(t)+\mathbf{F}_{\mathrm{St}}^{H}(t)+\mathbf{F}_{\mathrm{OS}}^{H}(t)-\rho\left\{6 \pi \Phi \cdot \Phi \cdot \Phi+\lim _{R \rightarrow \infty}\left(\int_{V_{f}(R)} \hat{\mathbf{M}}^{T} \cdot \hat{\mathbf{M}} d V-\frac{9 \pi}{2} \boldsymbol{\Phi} \cdot \boldsymbol{\Phi} R\right)\right\} \cdot \dot{\mathbf{U}}_{s}(t) \\
& -\rho^{*}\left\{\int_{V_{d}} \hat{\mathbf{M}}^{* T} \cdot \hat{\mathbf{M}}^{*} d V-V_{d} \mathbf{I}\right\} \cdot \dot{\mathbf{U}}_{s}(t)-\rho \lim _{R \rightarrow \infty} \int_{V_{f}(R)}\left(\mathbf{u}_{\mathrm{St}}^{\prime} \cdot \nabla \mathbf{u}_{\mathrm{St}}^{\prime}-\overline{\mathbf{U}}_{s} \cdot \nabla \mathbf{u}_{\mathrm{St}}^{\prime}\right) \cdot \hat{\mathbf{M}} d V \\
& -\rho^{*} \int_{V_{d}}\left(\mathbf{u}_{\mathrm{St}}^{* \prime} \cdot \nabla \mathbf{u}_{\mathrm{St}}^{* \prime}-\overline{\mathbf{U}}_{s} \cdot \nabla \mathbf{u}_{\mathrm{St}}^{* \prime}\right) \cdot \hat{\mathbf{M}}^{*} d V+o\left(\mu a U_{c} \operatorname{Re}\right)+o\left(\mu a U_{c} \frac{a^{2} v}{\tau_{c}}\right)
\end{aligned}
$$

where $V_{f}(R)$ represents a large spherical volume of radius $R$ with origin at the center of the drop, and $\mathbf{u}_{\mathrm{St}}^{\prime}$ and $\mathbf{u}_{\mathrm{St}}^{* \prime}$ are the steady Stokes solutions to the disturbance flow problem.

For a spherical drop the two terms in large curly braces from (61) combine to yield

$$
-6 \pi a^{3}\left\{\rho \frac{3 \lambda^{3}-3 \lambda-1}{27(\lambda+1)^{3}}+\rho^{*} \frac{1}{63(\lambda+1)^{2}}\right\} \cdot \dot{\mathbf{U}}_{s}(t) .
$$

This result agrees with the low-frequency (long-time) limit of the $O\left(\alpha^{2}\right)$ term from the result given by (57). This term is very different from the added mass in the high-frequency (short-time) limit, $-\frac{2}{3} \pi \rho a^{3} \dot{\mathbf{U}}_{s}(t)$, and reflects the uniqueness of the solid sphere which happens to have the same value in the low- and high-frequency limits. Recall that the contributions from the inertial terms inside the drop [the source of the second term of (62)] are identically zero for the case of a solid particle or bubble. Note also that since we have assumed the flow inside the drop is described by the steady Stokes equations to leading order in the longtime limit, we require that $\mu^{*}>\rho^{*} a U_{c}$. Thus, the second term of (62) does indeed go to zero in the limit of a bubble $\left(\mu^{*} \rightarrow 0\right)$ since $\rho^{*}$ must approach zero accordingly.

It can be seen in (61) that the long-time temporal response of the hydrodynamic force is dictated by the properties of $\mathbf{F}_{O \mathrm{~S}}^{H}$. This term, identified as the unsteady Oseen correction to the hydrodynamic force, was analyzed in LB. It was shown that its decay to steady state is algebraic for a step change from or to a zero velocity: behaving as $t^{-2}$ when the drop accelerates from rest and as $t^{-1}$ when it comes to rest. However, when the step changes are between finite velocities the ultimate decay of the hydrodynamic force is exponential. This contrast in temporal decay reflects the distinction between the creation (or destruction) of the wake structure, associated with the algebraic decay, and the modification of the wake structure already established, which leads to exponential decay. The fact that in all cases the temporal decay is faster than the $t^{-1 / 2}$ associated with unsteady Stokes flow reflects the efficient mechanism of convective transport of vorticity relative to that of diffusion. It should be reiterated that this behavior is observed on long time scales $\left(>v / U_{c}^{2}\right)$ and thus for small time the decay will go as $t^{-1 / 2}$.

\section{RESULTS AND DISCUSSION}

To provide some confirmation of the validity of the results we have obtained, we compare with the recently published numerical work of Mei and Klausner. ${ }^{12}$ They evaluated the force on a spherical bubble held fixed in a uniform flow with small fluctuations, a flow given by

$$
U^{\infty}(t)=U\left(1+\delta e^{-i \omega t}\right)
$$

with the condition $\delta<1$ and results for the drag evaluated to $O(\delta)$. By letting $U_{s}(t)=-U^{\infty}(t)$ in the force expres- 
sion for a bubble, (60), and with the aid of the frequency domain expression (57) and the use of MATHEMATICA to carry out the appropriate integrations, we arrive at the following expression for the force accurate to $O(\delta)$ and to $O(\operatorname{Re})$ :

$$
\begin{aligned}
\frac{F_{b}^{H}(t)}{6 \pi \mu a U}= & \frac{2}{3}+\frac{2}{3} \delta e^{-i \omega t}+\frac{1}{3} \alpha^{2} \delta e^{-i \omega t}+\frac{4}{9} \delta e^{-i \omega t}\left(\frac{\alpha}{1+\alpha / 3}-\alpha\right) \\
& +\frac{1}{6} \operatorname{Re}+\frac{4}{9} \operatorname{Re} \delta e^{-i \omega t} \frac{2^{1 / 2}(1-i)\left(\gamma_{\omega}+i\right)^{3 / 2}-2 i}{4 \gamma_{\omega}}
\end{aligned}
$$

The Reynolds number is defined by $\operatorname{Re}=a U / v$ and $\gamma_{\omega}=4 \omega v / U^{2}$ is a dimensionless low-frequency parameter. The first two terms originate from the pseudosteady Stokes drag, the third from the added mass and the acceleration of the imposed flow, and the fourth from the first two history integrals of (60): the unsteady Stokes history force less its low-frequency asymptote. The last two terms of (64) are from the unsteady Oseen correction, $\mathbf{F}_{\mathrm{Os}}^{H}$.

If we define the history force as the part of (64) that results from subtracting off both the finite zero-frequency components and the $O\left(\alpha^{2}\right)$ term $(\sim \omega)$, the dimensionless history force may be expressed as

$$
\begin{aligned}
\frac{F_{b h}^{H}(t)}{6 \pi \mu a U}= & \frac{4}{9} \delta e^{-i \omega t}\left(\frac{\alpha}{1+\alpha / 3}-\alpha\right) \\
& +\frac{4}{9} \operatorname{Re} \delta e^{-i \omega t}\left(\frac{2^{1 / 2}(1-i)\left(\gamma_{\omega}+i\right)^{3 / 2}-2 i}{4 \gamma_{\omega}}-\frac{3}{4}\right),
\end{aligned}
$$

where the $3 / 4$ term in (65) is necessary to remove the zero-frequency asymptote of the last term of (64). The real and imaginary parts of the history force coefficient have been evaluated numerically by Mei and Klausner ${ }^{12}$ for $\operatorname{Re}$ $=0.05,2.5$, and 20 . (Note that their definition of the Reynolds number is based on the bubble diameter, not the radius as is done here, so their values are reported as $0.1,5$, and 40.) Using the notation of Mei and Klausner, ${ }^{12}$ we define the following history force coefficients as a function of the frequency parameter $\epsilon=\left(\omega a^{2} / 2 v\right)^{1 / 2}$ :

$$
\begin{aligned}
D_{1 R H}(\epsilon)= & \operatorname{Real}\left[\frac{4}{9}\left(\frac{(-2 i)^{1 / 2} \epsilon}{1+(-2 i)^{1 / 2} \epsilon / 3}-(-2 i)^{1 / 2} \epsilon\right)\right. \\
& \left.+\frac{4}{9} \operatorname{Re}\left(\frac{2^{1 / 2}(1-i)\left(8 \epsilon^{2} / \mathrm{Re}^{2}+i\right)^{3 / 2}-2 i}{32 \epsilon^{2} / \mathrm{Re}^{2}}-\frac{3}{4}\right)\right],
\end{aligned}
$$

and

$$
\begin{aligned}
D_{1 I H}(\epsilon)= & \operatorname{Im}\left[\frac{4}{9}\left(\frac{(-2 i)^{1 / 2} \epsilon}{1+(-2 i)^{1 / 2} \epsilon / 3}-(-2 i)^{1 / 2} \epsilon\right)\right. \\
& \left.+\frac{4}{9} \operatorname{Re}\left(\frac{2^{1 / 2}(1-i)\left(8 \epsilon^{2} / \mathrm{Re}^{2}+i\right)^{3 / 2}-2 i}{32 \epsilon^{2} / \operatorname{Re}^{2}}-\frac{3}{4}\right)\right],
\end{aligned}
$$
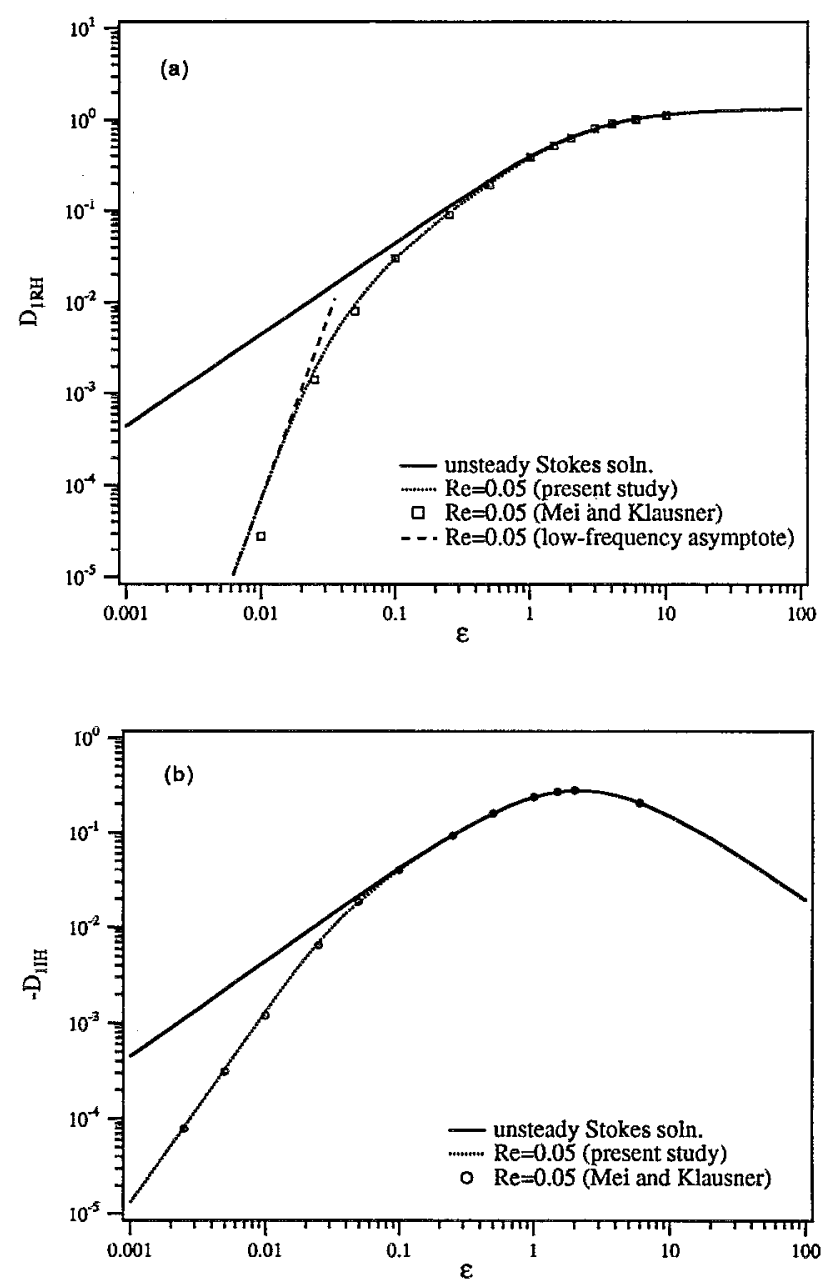

FIG. 1. Comparison of the (a) real and (b) imaginary parts of the history force coefficient for a bubble, $D_{1 R H}$ and $D_{1 I F}$, respectively, with the numerical results of Mei and Klausner ${ }^{12}$ as a function of the frequency parameter $\epsilon$.

where we have used the fact that $\alpha=(-2 i)^{1 / 2} \epsilon$ and $\gamma_{\omega}=8 \epsilon^{2} / \operatorname{Re}^{2}$. The case of $\operatorname{Re}=0.05$ is relevant to the current small Reynolds number study and is shown in Figs. 1 (a) and 1 (b) for the real and imaginary parts, $D_{1 R H}$ and $D_{1 I H}$, respectively. Also included is the unsteady Stokes result given by the first term of (66) and (67), which represents the asymptotic limit of the expressions as $R e \rightarrow 0$ for fixed $\epsilon$. The low-frequency asymptote in Fig. 1(a) is given by

$$
\lim _{\epsilon<\operatorname{Re}<1} D_{1 R H}(\epsilon)=\frac{8}{9} \frac{\epsilon^{4}}{\operatorname{Re}^{3}} .
$$

The results show very good agreement with the numerical work of Mei and Klausner over the entire range of frequency. This is consistent with the findings of a similar study by Lovalenti and Brady ${ }^{13}$ for solid spheres which showed good agreement with the numerical work of Mei et $a .^{14}$ up to $\operatorname{Re} \sim 0.5$.

Also in the analysis of Lovalenti and Brady ${ }^{13}$ is the inversion of the frequency-dependent result to the time domain for a general time-dependent motion, when the 
unsteady portion is small. A very similar derivation can be performed here. The interested reader is referred to the original work for the details. The result is

$$
\begin{aligned}
F_{b}^{H}(t)= & 6 \pi \mu a U\left(\frac{2}{3}+\frac{2}{3} U_{1}(t)+\frac{1}{6} \operatorname{Re}\left[1+2 U_{1}(t)\right]\right) \\
& +2 \pi \rho a^{3} \dot{U}_{1}(t)+8 \pi \mu a \int_{-\infty}^{t} e^{9 v(t-s) / a^{2}} \\
& \times \operatorname{erfc}\left(\sqrt{\frac{9 v(t-s)}{a^{2}}}\right) \dot{U}_{1}(s) d s \\
& -\frac{8 \pi \mu a}{\sqrt{\pi}} \int_{-\infty}^{t} \frac{\dot{U}_{1}(s)}{\sqrt{9 v(t-s) / a^{2}}} d s \\
& +6 \pi \mu a U \frac{2}{9} \frac{\operatorname{Re}}{\pi} \int_{-\infty}^{t} G(t-s) \dot{U}_{1}(s) d s,
\end{aligned}
$$

with

$$
G(t)=e^{-t U^{2} / 4 v} \int_{0}^{\infty} \frac{x^{3 / 2}}{(1+x)^{2}} e^{-x t U^{2} / 4 v} d x,
$$

where the imposed flow $U^{\infty}(t)=U\left[1+U_{1}(t)\right]$ has the condition $U_{1}(t)<1$ for all time.

Equations (66) and (67) also show good agreement at higher Reynolds number for high-frequency motion. This condition exists because the unsteady Stokes solution is valid even for moderate $\operatorname{Re}$ provided $\epsilon>\operatorname{Re}$. At low frequency $(\epsilon<\mathrm{Re})$, good qualitative agreement is achieved at higher Reynolds number only when the unsteady Oseen force is left by itself to predict the history force. The corrections from the unsteady Stokes solution [the terms contained in the first set of large parentheses in (66) and (67)], which are of higher order than $O(\mathrm{Re})$ at low frequency, are not properly matched because $(60)$ is strictly valid for very small $\mathrm{Re}$ and is only accurate to $O(\mathrm{Re})$. Although they are smaller than $O(\operatorname{Re})$, the unsteady Stokes corrections apparently erroneously alter the behavior of the history force at low frequency. This is not a problem for solid spheres because the unsteady Stokes history force is uniquely simple, being the same in both the high- and the low-frequency limit. The history force for a solid sphere can then be completely accounted for by the unsteady Oseen correction; there are no required unsteady Stokes corrections to the history force. This problem with bubbles (which also exists for drops) can be illustrated by noting that the unsteady Stokes corrections at low frequency contribute the quantity $-8 \epsilon^{3} / 81$ to $D_{1 R H}$. By comparison with (68), we see that it will actually dominate and incorrectly change the sign of $D_{1 R H}$ when $\epsilon<\operatorname{Re}^{3} / 9$, however, at that point $D_{1 R H}$ is less than $O\left(\operatorname{Re}^{9}\right)$. Thus, if one is studying low-frequency behavior at finite $\mathrm{Re}$, it is advisable to use only the unsteady Oseen correction to represent the history force for a bubble or drop.

Next, in order to show the variation of the force on a drop with drop properties and Reynolds number, we consider the history force on a spherical drop for the motion described by (63). Using the same arguments as for the bubble, the history force for a drop using (57) is given to $O(\delta)$ by

$$
\begin{aligned}
\frac{F_{d h}^{H}(t)}{6 \pi \mu a U}= & \delta e^{-i \omega t}\left(\frac{1 / 3}{\lambda+1}+\frac{2 / 3 \lambda+5 / 9}{(\lambda+1)^{2}} \alpha\right. \\
& \left.-\frac{(1+\alpha)^{2} f(\alpha \beta)}{\lambda g(\alpha \beta)+(3+\alpha) f(\alpha \beta)}\right) \\
& -\operatorname{Re} \delta e^{-i \omega t}\left(\frac{\lambda+2 / 3}{\lambda+1}\right)^{2} \\
& \times\left(\frac{2^{1 / 2}(1-i)\left(\gamma_{\omega}+i\right)^{3 / 2}-2 i}{4 \gamma_{\omega}}-\frac{3}{4}\right) .
\end{aligned}
$$

The history force coefficient can then be expressed as a function of $\alpha$ by

$$
\begin{aligned}
D_{1 H}(\alpha)= & \left(\frac{1 / 3}{\lambda+1}+\frac{2 / 3 \lambda+5 / 9}{(\lambda+1)^{2}} \alpha\right. \\
& \left.-\frac{(1+\alpha)^{2} f(\alpha \beta)}{\lambda g(\alpha \beta)+(3+\alpha) f(\alpha \beta)}\right) \\
& +\operatorname{Re}\left(\frac{\lambda+2 / 3}{\lambda+1}\right)^{2}\left(\frac{\left(4 \alpha^{2} / \operatorname{Re}^{2}+1\right)^{3 / 2}-1}{8 \alpha^{2} / \operatorname{Re}^{2}}-\frac{3}{4}\right),
\end{aligned}
$$

where we have used $\gamma_{\omega}=4 i \alpha^{2} / \operatorname{Re}^{2}$. The high-frequency behavior of (72) is given by

$$
\begin{aligned}
D_{1 H} \sim \alpha\left(1-\frac{\alpha}{3 \lambda}\right), \quad 1<\alpha \ll \frac{1}{\beta}, \lambda, \\
\sim \frac{3(2 / 3+\lambda)^{2}}{\lambda+1}-\frac{3}{4} \operatorname{Re}\left(\frac{\lambda+2 / 3}{\lambda+1}\right)^{2}, \quad 1, \lambda<\alpha<\frac{1}{\beta}, \\
\sim \frac{4}{3}-\frac{1}{3} \operatorname{Re}, \quad 1, \frac{1}{\beta} \ll \alpha \ll \frac{1}{\lambda \beta}, \\
\sim \frac{\lambda \beta}{\lambda \beta+1} \alpha, \quad \alpha \gg 1, \frac{1}{\beta}, \frac{1}{\lambda \beta},
\end{aligned}
$$

while the low-frequency behavior is

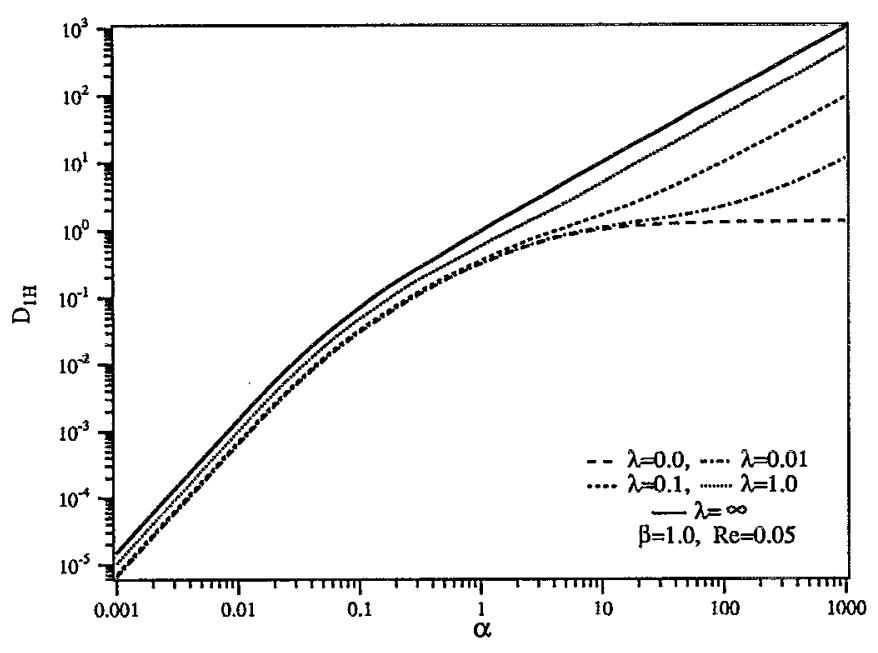

FIG. 2. Dependence of the history force coefficient $D_{1 H}$ for a drop on the viscosity ratio $\lambda$ as a function of the frequency parameter $\alpha$. 


$$
\begin{aligned}
D_{1 H} & \sim \frac{1 / 3}{\lambda+1}+\alpha-\frac{1}{\lambda \beta \alpha}-\frac{3}{4} \operatorname{Re}\left(\frac{\lambda+2 / 3}{\lambda+1}\right)^{2}, \quad \operatorname{Re}, \frac{1}{\beta}, \frac{1}{\lambda \beta} \ll \alpha<1, \\
& \sim \frac{4}{9} \alpha+\frac{\lambda \beta \alpha}{9}-\frac{3}{4} \operatorname{Re}\left(\frac{\lambda+2 / 3}{\lambda+1}\right)^{2}, \quad \operatorname{Re}, \frac{1}{\beta} \ll \alpha \ll 1, \frac{1}{\lambda \beta}, \\
& \sim\left(\frac{\lambda+2 / 3}{\lambda+1}\right)^{2} \alpha, \quad \operatorname{Re} \ll \alpha \ll 1, \frac{1}{\beta}, \\
& \sim \frac{3}{4} \operatorname{Re} \frac{\alpha^{2}}{\operatorname{Re}^{2}}\left(\frac{\lambda+2 / 3}{\lambda+1}\right)^{2}-\operatorname{Re}^{2} \frac{\alpha^{2}}{\operatorname{Re}^{2}} \frac{28+84 \lambda-3 \beta^{2} \lambda+63 \lambda^{2}-3 \beta^{2} \lambda^{2}}{189(1+\lambda)^{3}}, \quad \alpha \ll \frac{1}{\beta}, \operatorname{Re} .
\end{aligned}
$$

In Fig. 2 we show the dependence of $D_{1 H}$ on the viscosity ratio $\lambda$ for $\lambda=0$ to $\infty$. For low frequency, $\lambda$ alters the behavior of $D_{1 H}$ by simply a numerical coefficient. At high frequency, there is a stronger dependence on $\lambda$, particularly for small $\lambda$. This is because the properties of the drop are tending toward that of a bubble which has a very different high-frequency asymptote from that of a drop, $O(1)$ vs $O(\alpha)$. Figure 3 shows the dependence of $D_{1 H}$ on the kinematic viscosity ratio through the parameter $\beta$. In this case, since $\lambda$ is fixed, it actually shows the effect of the density of the drop relative to the surrounding fluid, i.e., $\beta \sim\left(\rho^{*} / \rho\right)^{1 / 2}$. There is little variation in $D_{1 H}$ with $\beta$ at low frequency. However, there is a stronger dependence, very similar to $\lambda$, at high frequency; higher/lower density drops behave similarly as higher/lower viscosity drops at high frequency.

In Fig. 4(a) the dependence of $D_{1 H}$ on the Reynolds number is shown. As the Reynolds number is increased the deviation of the history force from its corresponding unsteady Stokes solution increases, with the deviations becoming evident at higher frequencies. This deviation of the history force from the unsteady Stokes solution at low frequencies leads to a much different temporal behavior of the force on a bubble, drop, or particle at finite Reynolds num-

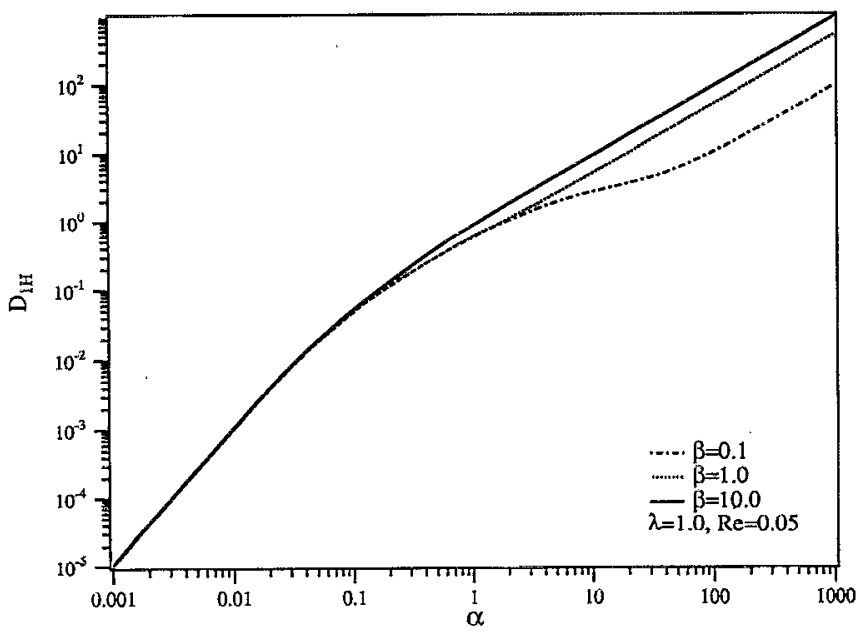

FIG. 3. Dependence of the history force coefficient $D_{1 A}$ for a drop on the kinematic viscosity ratio $\beta^{2}$ as a function of the frequency parameter $\alpha$. ber, particularly as steady state is approached. Finally, in Fig. 4(b) we plot the curves of Fig. 4(a) at finite Reynolds number in inertial coordinates rescaled by dividing by Re. It demonstrates that the results can be collapsed quite well on a single curve for given values of $\lambda$ and $\beta$. The rescaling works provided the high-frequency asymptote varies linearly with $\alpha$, e.g., we are not dealing with a bubble which shows no variation with $\alpha$ at high frequency, and it improves at intermediate $\alpha$ as $\lambda$ and $\beta$ are increased.

We now conclude by deriving the equation of motion for a bubble, drop, or particle in a fluid, appropriate for
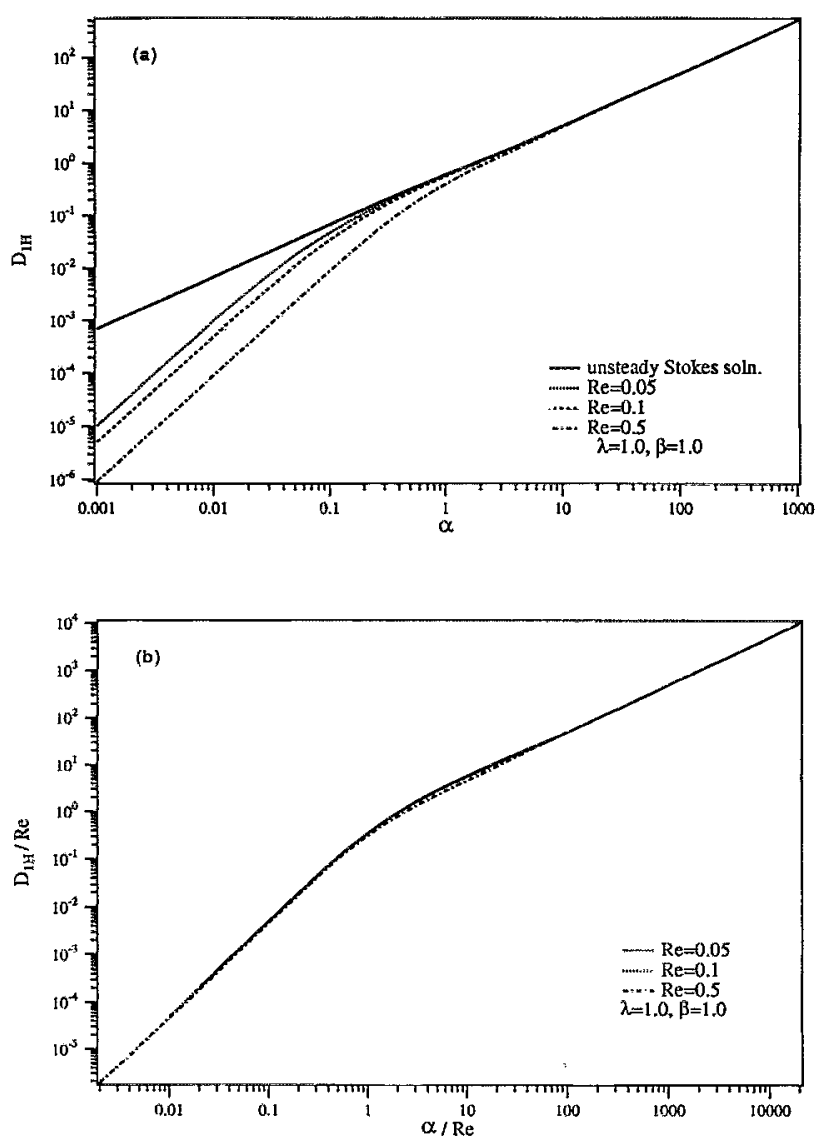

FIG. 4. Dependence of the history force coefficient $D_{1 H}$ for a drop on the Reynolds number $\operatorname{Re}$ as a function of the frequency parameter $\alpha$ : (a) viscous scale and (b) inertial scale. 
motion at small but finite Reynolds number. The general expression is given by

$$
m_{d} \dot{\mathbf{U}}=\mathbf{F}_{d}^{H}+\mathbf{F}^{\text {ext }},
$$

where $m_{d}$ is the mass of the body. Here, we have assumed that the only forces acting on the body are hydrodynamic forces and external body forces, $F^{\text {ext }}$, such as the buoyancy force due to gravity.

In the absence of velocity gradients in the imposed flow (the requirement here is that the Oseen time scale $v / U_{c}^{2}$ is much less than the characteristic shear rate in the imposed flow), the general expression for the hydrodynamic force is given by (51), where one can take into account the influence of the deformation of the body by including the last term of (26). The two volume integrals in (51) are absent for spherical bodies. For long time scale motion $\left(>a^{2} / v\right)$ of nonspheres they can be approximated using the solution of the steady Stokes flow field for the translating body. Under this condition, it can be shown that they contribute only a side force perpendicular to the direction of motion. ${ }^{1}$

For a spherical body the hydrodynamic force to $O(\mathrm{Re})$ simplifies to

$\mathbf{F}_{d}^{H}=\frac{4 \pi}{3} a^{3} \rho \dot{\mathbf{U}}^{\infty}(t)+\mathbf{F}_{U \mathrm{St}}^{H}-\sqrt{\frac{a^{2}}{\pi v}} \int_{-\infty}^{t} \frac{\dot{\mathbf{F}}_{\mathrm{St}}^{H}(s)}{\sqrt{t-s}} d s \cdot \boldsymbol{\Phi}+\mathbf{F}_{\mathrm{Os}}^{H}$,

where the unsteady Oseen force $\mathbf{F}_{\mathrm{Os}}^{H}$ is given by (55). A closed-form expression for the unsteady Stokes force $\mathbf{F}_{U S t}^{H}$ is available only for a solid body or a bubble. For bodies of intermediate viscosity one must invert the expression given by (57) to the time domain. The solution can be written formally as

$$
\begin{aligned}
\mathbf{F}_{U \mathrm{St}}^{H}(t)= & -3 \mu a \int_{-\infty}^{t} \overline{\mathbf{U}}_{s}(s) \int_{-\infty}^{\infty}\left(1+\alpha+\frac{a^{2}}{9}\right. \\
& \left.-\frac{(1+\alpha)^{2} f(\alpha \beta)}{\lambda g(\alpha \beta)+(3+\alpha) f(\alpha \beta)}\right) e^{-i \omega(t-s)} d \omega d s
\end{aligned}
$$

For a solid sphere (a body of infinite viscosity relative to the exterior fluid), this expression reduces to the wellknown result of Basset: ${ }^{15}$

$$
\begin{aligned}
\mathbf{F}_{U S \mathrm{St}}^{H}(t)= & -6 \pi \mu a \overline{\mathbf{U}}_{s}(t)-\frac{2}{3} \pi \rho a^{3} \overline{\mathrm{U}}_{s}(t) \\
& -6 \pi \mu a \sqrt{\frac{a^{2}}{\pi v}} \int_{-\infty}^{t} \frac{\overline{\mathbf{U}}_{s}(s)}{\sqrt{t-s}} d s .
\end{aligned}
$$

For an inviscid sphere it becomes ${ }^{11}$

$$
\begin{aligned}
\mathbf{F}_{U \mathrm{St}}^{H}(t)= & -4 \pi \mu a \overline{\mathbf{U}}_{s}(t)-\frac{2}{3} \pi \rho a^{3} \dot{\mathbf{U}}_{s}(t)-8 \pi \mu a \\
& \times \int_{-\infty}^{t} e^{9 v(t-s) / a^{2}} \operatorname{erfc}\left(\sqrt{\frac{9 v(t-s)}{a^{2}}}\right) \dot{\mathbf{U}}_{s}(s) d s .
\end{aligned}
$$

It is interesting to point out that in both of these limits the force expression is independent of the density of the material inside of the body. However, it can be seen in (77) that through the parameter $\beta$ the density of the drop can influence the force for general drop viscosities.

To compare the equation of motion for a solid sphere to that of an inviscid sphere (the distinction here from a bubble being that the body may have finite density), we combine the appropriate forms of $\mathbf{F}_{U \mathrm{St}}^{H}$ from (78) and (79) with $\mathbf{F}_{O \text { s }}^{H}$ from (55) in (76) to obtain (75) for a solid sphere:

$$
\begin{aligned}
& \left(m_{d}+\frac{1}{2} m_{f}\right) \overline{\mathbf{U}}_{s}+6 \pi \mu a \overline{\mathbf{U}}_{s}+6 \pi \mu a \frac{3}{8} \sqrt{\frac{a^{2}}{\pi v}}\left(\int _ { - \infty } ^ { t } \left\{\frac{2}{3} \overline{\mathbf{U}}_{s}^{\|}(t)-\left[\frac{1}{A^{2}}\left(\frac{\sqrt{\pi}}{2 A} \operatorname{erf}(A)-\exp \left(-A^{2}\right)\right)\right] \overline{\mathbf{U}}_{s}^{\|}(s)+\frac{2}{3} \overline{\mathbf{U}}_{s}^{1}(t)\right.\right. \\
& \left.\left.\quad-\left[\exp \left(-A^{2}\right)-\frac{1}{2 A^{2}}\left(\frac{\sqrt{\pi}}{2 A} \operatorname{erf}(A)-\exp \left(-A^{2}\right)\right)\right] \overline{\mathbf{U}}_{s}^{1}(s)\right\} \frac{2 d s}{(t-s)^{3 / 2}}\right) \\
& \quad=-\left(m_{d}-m_{f}\right) \dot{\mathbf{U}}^{\infty}(t)+\left(m_{d}-m_{f}\right) \mathbf{g},
\end{aligned}
$$

and for an inviscid sphere:

$$
\begin{aligned}
& \left(m_{d}+\frac{1}{2} m_{f}\right) \dot{\mathbf{U}}_{s}+4 \pi \mu a \overline{\mathbf{U}}_{s}+8 \pi \mu a \int_{-\infty}^{t} e^{9 v(t-s) / a^{2}} \operatorname{erfc}\left(\sqrt{\frac{9 v(t-s)}{a^{2}}}\right) \dot{\mathbf{U}}_{s}(s) d s-\frac{8 \pi \mu a}{\sqrt{\pi}} \int_{-\infty}^{t} \frac{\dot{U}_{s}(s)}{\sqrt{9 v(t-s) / a^{2}} d s} \\
& \quad+\pi \mu a \sqrt{\frac{a^{2}}{\pi v}}\left(\int _ { - \infty } ^ { t } \left\{\frac{2}{3} \overline{\mathbf{U}}_{s}^{\|}(t)-\left[\frac{1}{A^{2}}\left(\frac{\sqrt{\pi}}{2 A} \operatorname{erf}(A)-\exp \left(-A^{2}\right)\right)\right] \overline{\mathbf{U}}_{s}^{\|}(s)+\frac{2}{3} \overline{\mathbf{U}}_{s}^{\perp}(t)\right.\right. \\
& \left.\left.\quad-\left[\exp \left(-A^{2}\right)-\frac{1}{2 A^{2}}\left(\frac{\sqrt{\pi}}{2 A} \operatorname{erf}(A)-\exp \left(-A^{2}\right)\right)\right] \overline{\mathbf{U}}_{s}^{\perp}(s)\right\} \frac{2 d s}{(t-s)^{3 / 2}}\right) \\
& \quad=-\left(m_{d}-m_{f}\right) \dot{\mathbf{U}}^{\infty}(t)+\left(m_{d}-m_{f}\right) \mathbf{g},
\end{aligned}
$$


where $m_{f}$ is the mass of the exterior fluid displaced by the sphere and $\mathbf{g}$ is the acceleration due to gravity. Here, we have assumed the only external force is the buoyancy force, $\mathbf{F}^{\text {ext }}=\left(m_{d}-m_{f}\right) \mathrm{g}$.

Apart from the two additional history integrals in (81), the governing equations for the two bodies are essentially the same, in that they have the same terms with only a difference in numerical coefficients. For very short time scale motion $\left(<a^{2} / v\right)$, the temporal behavior of the two bodies will be nearly the same since the dominant contribution from the $1 \mathrm{hs}$ of their respective equations is the same first term. For time scales of $O\left(a^{2} / v\right)$, all terms of the governing equations will be important in the bodies' motion, and it is under this condition that the temporal behavior of the motion can possibly be different owing to the difference in the history dependence, a much weaker history dependence for the case of the inviscid drop. (We note this condition could have been observed from the results of unsteady Stokes flow without any of the Oseen-like considerations used here.) For long time scale motion $\left(>v / U_{c}^{2}\right)$, the temporal behavior of the bodies' motion will now be very similar since it is largely dictated by the unsteady Oseen force given by the last term on the lhs of (80) and (81), which differ only by a numerical coefficient.

${ }^{1}$ P. M. Lovalenti and J. F. Brady, "The hydrodynamic force on a rigid particle undergoing arbitrary time-dependent motion at small Reynolds number," to appear in J. Fluid Mech.
${ }^{2}$ R. F. Chisnell, “The unsteady motion of a drop moving vertically under gravity," J. Fluid Mech. 176, 443 (1987).

${ }^{3}$ T. D. Taylor and A. Acrivos, "On the deformation and drag of a falling viscous drop at low Reynolds number," J. Fluid Mech. 18, 466 (1964).

${ }^{4}$ L. G. Leal, Laminar Flow and Convective Transport Processes (Butterworth-Heinemann, Boston, 1992), pp. 197-229.

${ }^{5}$ L. G. Leal, "Particle motions in a viscous fluid," Annu. Rev. Fluid Mech. 12, 435 (1980).

${ }^{6} \mathrm{M}$. R. Maxey and J. J. Riley, "Equation of motion for a small rigid sphere in a nonuniform flow," Phys. Fluids 26, 883 (1983).

${ }^{7} \mathrm{C}$. W. Oseen, "Über die Stokes'sche Formel und über eine verwandte Aufgabe in der hydrodynamik," Ark. Mat. Astron. Fys. 6, 1 (1910).

${ }^{8} \mathrm{C}$. W. Oseen, "Über den Gültigkeitsbereich der Stokesschen Widerstandsformel," Ark. Mat. Astron. Fys. 9, 1 (1913).

${ }^{9}$ P. G. Saffman, "The lift on a small sphere in a slow shear flow," J. Fluid Mech. 22, 385 (1965).

${ }^{10}$ S. Kim and S. J. Karrila, Microhydrodynamics: Principles and Selected Applications (Butterworth-Heinemann, Boston, 1991), pp. 154-162.

${ }^{11} \mathrm{~S}$. Yang and L. G. Leal, "A note on memory-integral contributions to the force on an accelerating spherical drop at low Reynolds number," Phys. Fluids A 3, 1822 (1991).

${ }^{12}$ R. Mei and J. F. Klausner, "Unsteady force on a spherical bubble at finite Reynolds number with small fluctuations in the free-stream velocity," Phys. Fluids A 4, 63 (1992).

${ }^{13}$ P. M. Lovalenti and J. F. Brady, "The force on a sphere in a uniform flow with small amplitude oscillations at finite Reynolds number," to appear in J. Fluid Mech.

${ }^{14}$ R. Mei, C. J. Lawrence, and R. J. Adrian, "Unsteady drag on a sphere at finite Reynolds number with small fluctuations in the free-stream velocity," J. Fluid Mech. 233, 613 (1991).

${ }^{15}$ A. B. Basset, $A$ Treatise on Hydrodynamics (Cambridge: Deighton Bell, Cambridge, 1888), Vol. 2. 